\title{
Universal vs. risk-factor-based screening for gestational diabetes- an analysis from a 5-Year Portuguese Cohort
}

\author{
Claudia Matta-Coelho $\mathbb{1}^{1,2} \cdot$ Ana Margarida Monteiro ${ }^{1} \cdot$ Vera Fernandes $^{1} \cdot$ \\ Maria Lopes Pereira ${ }^{1}$ - Portuguese Diabetes and Pregnancy Study Group ${ }^{3}$. Selma B. Souto ${ }^{1}$
}

Received: 8 May 2018 / Accepted: 11 September 2018

(c) Springer Science+Business Media, LLC, part of Springer Nature 2018

\begin{abstract}
Purpose The criteria to screen for Gestational Diabetes Mellitus are not internationally consensual. In opposition to the universal screening performed in Portugal, certain countries advocate a risk-factor-based screening. We aim to compare obstetric and neonatal outcomes in pregnant women with and without risk factors treated for Gestational Diabetes Mellitus. Methods Retrospective and multicentric study of 12,006 pregnant women diagnosed with Gestational Diabetes Mellitus between 2011 and 2015, in Portugal. Gestational Diabetes Mellitus was diagnosed according to the International Association of the Diabetes and Pregnancy Study Groups criteria. Risk factors: body mass index $>30 \mathrm{~kg} / \mathrm{m}^{2}$, history of Gestational Diabetes Mellitus, history of macrossomic newborn (birth weight $>4000 \mathrm{~g}$ ) or first-degree relatives with Type 2 Diabetes Mellitus. Exclusion criteria: lack of data concerning risk factors $(n=1563)$.

Results At least one risk factor was found in $68.2 \%(n=7123)$ pregnant women. Pregnant women with risk factors were more frequently medicated with insulin $(p<0.001)$, caesarean section was more commonly performed $(p<0.001)$, their newborns were more frequently large-for-gestational-age $(p<0.001)$ and neonatal morbidity was higher $(p=0.040)$ in comparison to pregnant women without risk factors. The Diabetes Mellitus reclassification test showed an increased frequency of intermediate hyperglycaemia and Diabetes Mellitus in women with risk factors $(p<0.001)$.

Conclusion Almost one-third of pregnant women would have remained undiagnosed if risk-based-factor screening were implemented in Portugal. Women without risk factors presented fewer obstetric and neonatal complications. However, more than one third required insulin therapy.
\end{abstract}

Keywords Diabetes gestational $\cdot$ Risk factors $\cdot$ Diagnosis $\cdot$ Mass screening

\section{Introduction}

Gestational Diabetes Mellitus (GDM) is defined as a subtype of abnormal glucose tolerance resulting in hyperglycaemia of variable severity with onset during pregnancy. It is associated with an increased risk of adverse maternal, foetal and neonatal outcomes [1]. In Portugal, in 2015, the prevalence of GDM was $7.2 \%$ [2]. The criteria to screen for GDM are not internationally consensual [3]. The World

Claudia Matta-Coelho

claudiadmcoelho@gmail.com

1 Endocrinology Department, Hospital de Braga, Braga, Portugal

2 Center for Health Technology and Services Research (CINTESIS), University of Porto, Porto, Portugal

3 Portuguese Society of Diabetology, Porto, Portugal
Health Organization (WHO) recommended that local authorities ought to decide on the screening coverage depending on local resources and priorities [4]. In Portugal, screening for GDM is offered to all pregnant women [5]. However, in certain countries, like the United Kingdom, the screening is risk-factor-based [6]. Universal screening allows higher GDM detection rates, but more women are subjected to inconvenient testing, there is additional pregnancy medical intervention with increased health care costs and further maternal anxiety [7]. Besides, it has been suggested that the additional cases detected exhibit a milder to moderate hyperglycaemia [8] and might have less clinical significance [9]. Currently, the scientific evidence available is insufficient to favour a screening method [7]. The National Institute for Health and Care Excellence (NICE) clinical practice recommendations consider that the costeffectiveness ratios do not support universal screening [6]. The diagnostic criteria for GDM of the International 
Association of the Diabetes and Pregnancy Study Group (IADPSG) [10] is based on the Hyperglycaemia and Adverse Pregnancy Outcome study [11]. These criteria were implemented in Portugal in 2011, lowering the threshold for GDM and subsequently more women have been diagnosed with GDM [2]. Furthermore, more women with GDM are expected due to the obesity epidemic and increased maternal age [12], straining the Portuguese National Health System.

We hypothesised that pregnant women with risk factors (RF) presented worse obstetric and neonatal outcomes compared to women without RF. Thus, we aim to compare obstetric and neonatal outcomes between these two groups, and therefore reflect about risk-factor-based vs. universal screening for GDM.

\section{Materials and methods}

\section{Data sources and collection}

This is a retrospective cohort study of 12,006 pregnant women based on data from the Portuguese National Registry of Gestational Diabetes. The register is organized by the Portuguese Diabetes and Pregnancy Study Group of the Portuguese Society of Diabetology. The data is collected from 28 hospitals from all regions of Portugal and only includes pregnant women with GDM. The register is voluntary. We included pregnant women with GDM that gave birth from 2011 to 2015 . The medical team obtained information about the pregnant women with GDM since the first consultation until the Diabetes Mellitus (DM) reclassification test. The data was anonymised and none of the authors had access to patient identification. We excluded 1563 women due to a lack of data regarding RF. The final sample was constituted by 10,443 pregnant women.

\section{Definitions}

The diagnosis of GDM, according to IADPSG, is established by fasting plasma glucose (FPG) at the first pre-natal visit, between the 11th and 13th week plus 6 days gestational age, or by a $2 \mathrm{~h} 75-\mathrm{g}$ oral glucose tolerance test (OGTT) performed between 24 and 28 weeks of gestation [10]. The diagnostic criteria for GDM are FPG between 92 and $125 \mathrm{mg} / \mathrm{dL}$, or plasma glucose $92-125 \mathrm{mg} / \mathrm{dL}$, superior to $180 \mathrm{mg} / \mathrm{dL}$ or $153-199 \mathrm{mg} / \mathrm{dL}$ at 0,60 , or $120 \mathrm{~min}$, respectively, with the $2 \mathrm{~h} \mathrm{75-g} \mathrm{OGTT} \mathrm{[5].} \mathrm{All} \mathrm{pregnant}$ women diagnosed with GDM were referred to a specialized diabetes care team including a diabetologist, an obstetrician, a dietician, and a nurse. They were instructed to evaluate capillary glycaemia four times a day, at fasting and 1-h after the beginning of the three main meals. The pregnant women with GDM were seen by the diabetologist every 1-4 weeks. A personalised diet was recommended to all women. The glycaemic targets defined were less than $90 \mathrm{mg} / \mathrm{dL}$ at fasting and less than $120 \mathrm{mg} / \mathrm{dL}$ 1-h after meals. Pharmaceutical intervention with metformin or insulin was initiated if these glycaemic targets were not accomplished with nutritional therapy and physical activity after one or two weeks. Metabolic control and further therapeutic adjustments were assessed by glycaemic patterns, ultra-sound foetal development, and amniotic fluid changes. The newborns were classified as large-for-gestational-age (LGA) and small-forgestational-age (SGA) if their weight for gestational age was superior to 90th percentile (P90) or inferior to 10th percentile (P10) respectively, according to Fenton Growth charts. Neonatal morbidity comprised neonatal hypoglycaemia, neonatal hyperbilirubinemia, respiratory distress syndrome, congenital anomalies, and intensive care unit hospitalisation. We considered RF in light of NICE guidelines recommendations: body mass index $(\mathrm{BMI})>30 \mathrm{~kg} / \mathrm{m}^{2}$ (obesity), history of GDM, history of macrossomic newborn (birth weight $>4000 \mathrm{~g}$ ) or first-degree relatives with Type 2 Diabetes Mellitus (T2DM). The criteria about ethnic family origin was not considered. The type of delivery was divided into eutocic, vaginal instrumented delivery (forceps and ventouse) and caesarean. Six to eight weeks after delivery, all women were invited to undergo the DM reclassification test with $2 \mathrm{~h}$ 75-g OGTT. According to the American Diabetes Association (ADA), the diagnostic criteria for DM, impaired glucose tolerance (IGT) and impaired fasting glucose is $\mathrm{FPG} \geq 126 \mathrm{mg} / \mathrm{dL}$ or 2 -h plasma glucose $\geq 200 \mathrm{mg} / \mathrm{dL}$, 2-h plasma glucose between 140 and $199 \mathrm{mg} / \mathrm{dL}$ and a FPG between 100 and $125 \mathrm{mg} / \mathrm{dL}$, respectively.

\section{Statistical analysis}

Normal continuous variables were described by the mean and standard deviation. Non-normal continuous variables were described by the median and interquartile range (IQR). The normality was verified by the observation of histograms. Categorical variables were described by the absolute and relative frequencies. To compare continuous variables between two independent groups we used the Student $t$-test (normal distributions) or Mann-Whitney $U$-test (non-normal distributions). Chi-square test $\left(\chi^{2}\right)$ was applied to analyse independence between two categorical variables. Univariate and multivariate logistic regression were performed to study the association of each risk factor with the caesarean. Values of $p \leq 0.05$ were considered statistically significant. Data analysis was performed using IBM SPSS $^{\mathrm{TM}}$ software version 25. 
Table 1 General characteristics of the total cohort and of pregnant women with and without RF

\begin{tabular}{lllll}
\hline & Without RF & With RF & Total cohort & $p$-value \\
\hline Age, years mean $( \pm \mathrm{SD})$ & $32.6(5.4)$ & $33.5(5.2)$ & $33.2(5.3)$ & $0.001^{\mathrm{a}}$ \\
Non-portuguese & 14.2 & 11 & 12 & $0.001^{\mathrm{b}}$ \\
$\%(n / N)$ & $(361 / 2547)$ & $(615 / 5569)$ & $(976 / 8116)$ & \\
Pregravid BMI $\left(\mathrm{kg} / \mathrm{m}^{2}\right)$ & 23.7 & 28.3 & 26 & $0.001^{\mathrm{c}}$ \\
Median $(\mathrm{IQR})$ & $(21.7-26.3)$ & $(24-33.1)$ & $(22.9-30.9)$ & \\
1 st degree relatives with T2DM $(\%)$ & 0 & 71.8 & 48.5 & $0.001^{\mathrm{b}}$ \\
$(n / N)$ & $0 / 3320$ & $(4966 / 6912)$ & $(4966 / 10232)$ & \\
Previous foetal macrosomia & 0 & 9.2 & 6.1 & $0.001^{\mathrm{b}}$ \\
$\%(n / N)$ & $0 / 3320$ & $(589 / 6368)$ & $(589 / 9688)$ & \\
Previous GDM & 0 & 21.1 & 13.9 & $0.001^{\mathrm{b}}$ \\
$\%(n / N)$ & $0 / 3320$ & $(1354 / 6404)$ & $(1354 / 9724)$ & \\
Parity, $n$ & 0 & 1 & 1 & $0.001^{\mathrm{c}}$ \\
Median $(\mathrm{IQR})$ & $(0-1)$ & $(0-1)$ & $(0-1)$ & \\
\hline
\end{tabular}

$S D$ standard deviation, $I Q R$ interquartil range, $B M I$ body mass index, $T 2 D M$ type 2 diabetes mellitus, $G D M$ gestational diabetes mellitus

${ }^{\mathrm{a}} t$-test

${ }^{\mathrm{b}} \mathrm{Chi}$-square test

${ }^{\mathrm{c}}$ Mann-Whitney test

\section{Results}

We analysed data from 10,443 women, with a mean age of $33.2 \pm 5.3$ years old, of which $68.2 \%(n=7123)$ had at least one RF. The most common RF was first-degree relatives with T2DM $(71.8 \% ; n=4966 / 6912)$, followed by obesity (45.9\%; $n=3267 / 7123)$, history of GDM $(21.1 \% ; n=$ $1354 / 6404)$ and history of foetal macrosomia $(9.2 \% ; n=$ 589/6368). From 2011 to 2014, no difference in the proportion of pregnant women with RF was observed. However, in 2015 there was an increase in this group compared to previous years $(75.1 \%$ vs. $66.2 \% ; p<0.001)$.

Women without RF were younger than those with RF (32.6 years vs. 33.5 years; $p<0.001$ ). The detailed characteristics of women with GDM are described in Table 1.

The diagnosis of GDM in both groups was more frequently established by OGTT at the 24-28th gestational week than fasting glycaemia at the first pre-natal visit (60.6 vs. $39.4 \% ; p<0.001)$. Nevertheless, women with RF were more frequently diagnosed by fasting glycaemia at the first pre-natal visit in comparison to women without RF (40.2 vs. $37.7 \% ; p=0.015)$. The delay from diagnosis to the first consultation with the specialized diabetes care team was 3 weeks (IQR 2.0-6.0) and was similar between groups ( $p$ $=0.93$ ). In the pregnant women without RF vs. with RF, insulin therapy was less frequently initiated (38.4 vs. $48.6 \% ; p<0.001)$, the starting week of therapy was deferred one week (30th gestational week vs. 29th gestational week; $p<0.001)$ and total daily doses of insulin were inferior (14 insulin units vs. 18 insulin units; $p<0.001)$. Moreover, treatment with metformin was less frequently used $(5.6$ vs. $10.2 \% ; p<0.001)$. Regarding obstetric complications, pre-eclampsia occurred more frequently in women with RF (3.4 vs. $2.4 \% ; p=0.003$ ), whereas no differences in hydramnios or foetal death were observed. Caesarean section was more commonly performed in women with RF (38.4 vs. $30.6 \% ; p<0.001$ ), although vaginal instrumented deliveries were more frequent in women without RF (18.2 vs. $14.0 \% ; p<0.001)$. No differences occurred in eutocic births, in urgent or programmed caesareans, nor gestational age. The detailed characteristics of the obstetric outcomes are described in Table 2.

The pregnant women with first degree relatives with T2DM, previous foetal macrosomia and a BMI $>30 \mathrm{~kg} / \mathrm{m}^{2}$ presented an increased risk for caesarean section compared to pregnant women without these risk factors $(p<0.001)$. When adjusting these variables to each other the chance of caesarean section remained elevated $(p<0.001)$. The univariate and multivariate logistic regression are shown in Tables 3 and 4.

The prevalence of LGA newborns was higher in the group of pregnant women with RF (5.1 vs. $2.3 \%$; $p<$ 0.001 ), while pregnant women without RF gave birth more frequently to SGA newborns (14.6 vs. $12.3 \% ; p<0.001)$. The neonatal morbidity was higher in the newborns of pregnant women with RF (19.3 vs. $17.5 \% ; p=0.040)$. No differences in neonatal deaths were observed. Neonatal outcomes are described in Table 5.

The DM reclassification test showed an increased frequency of impaired fasting glycaemia, impaired glucose tolerance and diabetes in women with RF (8.6\% vs. $5.2 \%$; $p<0.001$ ). 
Table 2 Obstetric outcomes of the total cohort and of pregnant women with and without RF

\begin{tabular}{|c|c|c|c|c|}
\hline & Without RF & With RF & Total cohort & $p$-value \\
\hline Gestational hypertension & 7.2 & 12.7 & 10.9 & $0.001^{\mathrm{a}}$ \\
\hline$\%(\mathrm{n} / \mathrm{N})$ & $(226 / 3157)$ & $(841 / 6626)$ & $(1067 / 9783)$ & \\
\hline Hydramnios & 2.8 & 3.2 & 3.1 & $0.126^{\mathrm{a}}$ \\
\hline$\%(\mathrm{n} / \mathrm{N})$ & $(88 / 3711)$ & $(213 / 6616)$ & $(301 / 9793)$ & \\
\hline Pre-eclampsia & 2.4 & 3.4 & 3 & $0.003^{\mathrm{a}}$ \\
\hline$\%(n / N)$ & $(74 / 3142)$ & $(223 / 6613)$ & (297/9755) & \\
\hline Weight gain, $\mathrm{kg}$ (mean $\pm \mathrm{SD})$ & $10.9(5.4)$ & $9.1(6.2)$ & $9.7(6)$ & $0.001^{\mathrm{b}}$ \\
\hline Therapy with insulin \% (n) & $38(1256)$ & $49(3441)$ & $45.4(4697)$ & $0.001^{\mathrm{a}}$ \\
\hline Foetal death & 0.3 & 0.4 & 0.3 & $0.285^{\mathrm{a}}$ \\
\hline$\%(n / N)$ & $(8 / 3092)$ & $(24 / 6772)$ & (32/9864) & \\
\hline Gestational age, weeks median (IQR) & $39(38-39)$ & $39(38-39)$ & $39(38-39)$ & $0.101^{\mathrm{c}}$ \\
\hline Induced delivery & 35.5 & 40.2 & 38.9 & $0.001^{\mathrm{a}}$ \\
\hline$\%(n / N)$ & $(319 / 899)$ & $(941 / 2343)$ & $(1260 / 3242)$ & \\
\hline \multicolumn{5}{|l|}{ Caesarean } \\
\hline$\%(n / N)$ & 47.2 & 46.9 & 47 & \\
\hline Urgent & $(427 / 905)$ & $(1133 / 2417)$ & $(1560 / 3322)$ & $0.453^{\mathrm{a}}$ \\
\hline \multirow[t]{2}{*}{ Elective } & 52.8 & 53.1 & 53 & \\
\hline & $(478 / 905)$ & $(1284 / 2417)$ & $(1762 / 3322)$ & \\
\hline \multicolumn{5}{|l|}{ Type of delivery \% ( $n$ ) } \\
\hline Eutocic & $51.3(1632)$ & $47.6(3223)$ & $48.8(4855)$ & $0.078^{\mathrm{a}}$ \\
\hline Vaginal instrumented delivery & $18.2(578)$ & $14(946)$ & $15.3(1524)$ & $0.001^{\mathrm{a}}$ \\
\hline Caesarian & $30.6(973)$ & $38.4(2602)$ & $35.9(3575)$ & $0.001^{\mathrm{a}}$ \\
\hline
\end{tabular}

$S D$ standard deviation, $I Q R$ interquartil range

${ }^{\mathrm{a} C h i}$-square test; Mann-Whitney test

${ }^{\mathrm{b}} t$-test
Table 3 Univariate logistic regression

\begin{tabular}{|c|c|c|}
\hline Variables & OR $(95 \% \mathrm{CI})$ & $p$-value \\
\hline \multicolumn{3}{|c|}{ 1st degree relatives with $T 2 D M$} \\
\hline No & Reference & - \\
\hline Yes & $1.248(1.149-1.356)$ & $<0.001$ \\
\hline \multicolumn{3}{|c|}{ Previous pregnancy with GDM } \\
\hline No & Reference & - \\
\hline Yes & $1.099(0.973-1.242)$ & 0.130 \\
\hline \multicolumn{3}{|c|}{ Previous foetal macrosomia } \\
\hline No & Reference & - \\
\hline Yes & $1.665(1.403-1.977)$ & $<0.001$ \\
\hline BMI & $1.036(1.029-1.043)$ & $<0.001$ \\
\hline
\end{tabular}

Dependent variable: caesarean

$T 2 D M$ type 2 diabetes mellitus, GDM gestational diabetes mellitus, $B M I$ body mass index

\section{Discussion}

\section{Universal screening vs. risk-factor based}

The IADPSG criteria used in Portugal for GDM screening is currently recommended by the International Federation of Gynaecology and Obstetrics and most international organizations, with some exceptions namely the American College of Obstetricians and Gynaecologists and NICE.
Table 4 Multivariate logistic regression

\begin{tabular}{|c|c|c|}
\hline Variables: & OR $(95 \% \mathrm{CI})$ & $p$-value \\
\hline \multicolumn{3}{|c|}{ 1st degree relatives with $T 2 D M$} \\
\hline No & Reference & - \\
\hline Yes & $1.203(1.101-1.314)$ & $<0.001$ \\
\hline \multicolumn{3}{|c|}{ Previous foetal macrosomia } \\
\hline No & Reference & - \\
\hline Yes & $1.468(1.223-1.761)$ & $<0.001$ \\
\hline BMI & $1.035(1.027-1.043)$ & $<0.001$ \\
\hline
\end{tabular}

Dependent variable: caesarean

$T 2 D M$ type 2 diabetes mellitus, $G D M$ gestational diabetes mellitus, $B M I$ body mass index

One of the limitations of risk-factor-based screening is the difficulty to ascertain the adequate RF for each population. Interestingly, in our study, the frequency of pregnant women with at least one RF (68.2\%) was higher in comparison to other studies [13]. However, if risk-basedfactor screening ought to be implemented in Portugal almost a third of pregnant women would have remained undiagnosed. Another limitation of risk-factor-based screening is the fact that previous history of GDM and macrosomia are considered as risk factors, so primiparous that may develop GDM complications during the first pregnancy are excluded. Additionally, age, which is known to be a traditional RF for GDM, is omitted in NICE 
Table 5 Neonatal outcomes of the total cohort and of pregnant women with and without RF

\begin{tabular}{lllll}
\hline & Without RF & With RF & Total cohort & $p$-value \\
\hline Neonatal morbidity & 17.5 & 19.3 & 18.7 & $0.021^{\mathrm{b}}$ \\
$\%(n / N)$ & $(525 / 2993)$ & $(1216 / 6293)$ & $(1741 / 9286)$ & \\
Neonatal hypoglycaemia & 3.2 & 4 & 3.7 & $0.024^{\mathrm{b}}$ \\
$\%(n / N)$ & $(93 / 2941)$ & $(248 / 6174)$ & $(341 / 9115)$ & \\
Neonatal Hyperbilirubinemia & 9.9 & 11.6 & 11 & $0.007^{\mathrm{b}}$ \\
$\%(n / N)$ & $(293 / 2966)$ & $(723 / 6235)$ & $(1016 / 9201)$ & \\
Respiratory distress syndrome \% $(n / N)$ & $2(59 / 2960)$ & $2.8(176 / 6224)$ & $2.6(235 / 9184)$ & $0.019^{\mathrm{b}}$ \\
Weight of newborn, kg Median $(\mathrm{IQR})$ & $3110(2820-3380)$ & $3180(2870-3480)$ & $3160(2855-3450)$ & $0.001^{\mathrm{c}}$ \\
Large for gestational age \% $(n / N)$ & $2.3(69 / 3061)$ & $5.1(335 / 6535)$ & $4.2(404 / 9596)$ & $0.001^{\mathrm{b}}$ \\
Small for gestational age $\%(n / N)$ & $14.6(448 / 3061)$ & $12.3(803 / 6535)$ & $13(1251 / 9596)$ & $0.001^{\mathrm{b}}$ \\
Adequate for gestational age \% $(n / N)$ & $83.1(2544 / 3061)$ & $82.6(5397 / 6535)$ & $82.2(7941 / 9596)$ & $0.065^{\mathrm{b}}$ \\
Admission to intensive care unit \% $(n / N)$ & $5(149 / 2972)$ & $6(375 / 6239)$ & $5.7(524 / 9211)$ & $0.054^{\mathrm{b}}$ \\
Congenital anomalies \% $(n / N)$ & $2.2(62 / 2832)$ & $3.4(204 / 6040)$ & $3(266 / 8872)$ & $0.001^{\mathrm{b}}$ \\
Neonatal death \% $(n / N)$ & $0.4(8 / 2037)$ & $0.2(11 / 4709)$ & $0.3(19 / 6746)$ & $0.187^{\mathrm{b}}$ \\
\hline
\end{tabular}

$S D$ standard deviation, $I Q R$ interquartil range

${ }^{\mathrm{a}} t$-test

${ }^{\mathrm{b}} \mathrm{Chi}$-square test

${ }^{c}$ Mann-Whitney test screening criteria [14]. Risk-factor-based screening is also more complex than universal screening and requires more attention to previous obstetric history by health professionals, which may result in inadequate screening. The main goal of risk-factor-based screening is to recognise pregnant women at greater risk of complications who would most gain from intensive management, while other pregnant women would be relieved from the burden of further medical interventions. A more rational use of resources would allow, for instance, a reduction of time from diagnosis to the first consultation that in our study was three weeks. Considering that the diagnosis of GDM is mainly established after the 24th week, a 3-week gap until observation and management by the specialized diabetes care team is relevant.

\section{Diagnosis and treatment}

The increased frequency of GDM diagnosis by fasting plasma glycaemia and the increased incidence of prediabetes and DM at the reclassification test suggest that women with RF suffer a more severe form of abnormal glucose metabolism. However, we emphasise that the diagnosis by fasting plasma glycaemia is not consensual and is not recommended by ADA [15]. Our results showed that women without RF required less frequently therapy with insulin and in a lower dosage. Yet, still, 38\% had inadequate metabolic control and initiated insulin therapy. In 2016, a new consensus for GDM treatment was introduced in Portugal and the glycaemic targets were modified to $95 \mathrm{mg} / \mathrm{dL}$ when fasting and $140 \mathrm{mg} / \mathrm{dL}$ one hour after the meal, instead of 90 and $120 \mathrm{mg} / \mathrm{dL}$ in use at the time of this study. Therefore, the proportion of women with GDM in need of pharmacotherapy is expected to decrease.

\section{Obstetric/Neonatal outcomes}

The label of "GDM" may prompt higher rates of caesarean section rather than clinical indication [16-18]. On the other hand, Farrar et al. showed that after the transition from riskfactor-based to universal screening and subsequently higher GDM detection rates, caesarean section rates did not rise in their population. However, there was an increase in labour induction [8]. The prevalence of LGA in our cohort was lower than in other studies [19]. One of the possible explanations for this is the use of the Fenton curves for neonatal assessment, that like shown by other authors may underestimate LGA in comparison with other curves [20]. Additionally, these findings are probably related to the adequate glycaemic control attained in both groups. Nevertheless, there were significant differences in LGA between the two groups, strengthening our hypothesis. Our findings showed a high frequency of SGA in the whole cohort and even higher in the group without RF. One of the main goals of the management of women with GDM is to control foetal growth, but tight glucose control may be associated with a higher incidence of SGA and adverse neonatal outcomes [21]. Thus, it is important to avoid overmedicalization of GDM, which we think has been considered in the new glycaemic targets proposed for GDM since 2016.

\section{Reclassification test}

The DM reclassification test revealed a higher prevalence of abnormal glucose metabolism in women with RF. However, of the women without any known RF, there were 5\% that also had an abnormal result. These women have a higher risk of T2DM in the future, despite being young, non-obese 
and with no family history of DM. This is a further concern about selective screening in GDM, as these women would not be identified and there would be no opportunity to intervene longer-term to promote enhanced screening, lifestyle change or early diagnosis of T2DM in the future.

\section{Strengths/Limitations}

The strengths of our study are the sample size obtained from several hospitals in all regions in Portugal and by addressing an issue with potential implications on current measures.

The limitations are related to the retrospective nature of the study, loss of data due to incomplete fill of the database, and the international heterogeneity in screening and diagnostic criteria for GDM troubling comparisons between studies. In addition, both groups were treated and the better outcomes observed in the group without RF must be cautiously interpreted. Ideally, comparisons should be established between women diagnosed with GDM with no RF and pregnant women glucose-tolerant in the Portuguese population.

Nevertheless, according to current measures, additional attention is required for pregnant women with GDM and RF.

\section{Conclusion}

Pregnant women with RF diagnosed with GDM on universal screening had worse obstetric and neonatal outcomes than those without RF.

\section{Compliance with ethical standards}

Conflict of interest The authors declare that they have no conflict of interest.

Ethical approval All procedures performed in studies involving human participants were in accordance with the ethical standards of the institutional and/or national research committee and with the 1964 Helsinki declaration and its later amendments or comparable ethical standards. For this type of study formal consent is not required.

\section{References}

1. B. Baz, J.P. Riveline, J.F. Gautier, Gestational diabetes mellitus: definition, aetiological and clinical aspects. Eur. J. Endocrinol. 174, 43-51 (2016)

2. Diabetologia S. P. Factos e números. Observatório Nacional da Diabetes. http://www.spd.pt/images/bolsas/dfn2015.pdf (2016). Accessed 1 Sept 2017
3. H. Berger, M. Sermer, Counterpoint: selective screening for gestational diabetes mellitus. Diabetes Care 32, 1352-1354 (2009)

4. World Health Organization. Diagnostic criteria and classification of hyperglycaemia first detected in pregnancy. WHO. http://www. who.int/diabetes/publications/Hyperglycaemia_In_Pregnancy/en/ (2013). Accessed 1 Sept 2017

5. Diabetologia SP. Consenso "Diabetes Gestacional" - Atualização 2016. Portuguese Diabetes and Pregnancy Study Group. http://www.spd.pt/images/consenso_dg_atualizacao2016.pdf (2016). Accessed 1 Sept 2017

6. National Institute for Health and Care Excellence. Diabetes in pregnancy: management from preconception to the postnatal period. https://www.nice.org.uk/guidance/ng3 (2015). Accessed 1 Sept 2017

7. J. Tieu, A. J. McPhee, C. A. Crowther, P. Middleton, E. Shepherd, Screening for gestational diabetes mellitus based on different risk profiles and settings for improving maternal and infant health. Cochrane Database Syst Rev. 8, 1-67 (2017).

8. D. Farrar, L. Fairley, J. Wright, D. Tuffnell, D. Whitelaw, D.A. Lawlor, Evaluation of the impact of universal testing for gestational diabetes mellitus on maternal and neonatal health outcomes: a retrospective analysis. BMC Pregnancy Childbirth 14, 1-8 (2014)

9. D. Naylor, M. Sermer, E. Chen, D. Farine, Selective screening for gestational diabetes mellitus. N. Engl. J. Med. 337, 1591-1596 (1997)

10. International Association of Diabetes and Pregnancy Study Groups Consensus Panel., International Association of Diabetes and Pregnancy Study Groups recommendations on the diagnosis and classification of hyperglycemia in pregnancy. Diabetes Care 33, 676-682 (2010)

11. The HAPO Study Cooperative Research Group., Hyperglycemia and adverse pregnancy outcomes. N. Engl. J. Med. 358, 1991-2002 (2008)

12. G.E. Avalos, L.A. Owens, F. Dunne, Applying current screening tools for gestational diabetes mellitus to a european population: Is it time for change? Diabetes Care 36, 3040-3044 (2013)

13. Adam S., Rheeder P. Screening for gestational diabetes mellitus in a South African population: prevalence, comparison of diagnostic criteria and the role of risk factors. 107, 523-527 (2017)

14. F. Galtier, Definition, epidemiology, risk factors. Diabetes Metab. 36, 628-651 (2010)

15. American Diabetes Association. Standards of Medical Care in Diabetes 2017. Diabetes Care. 40, 1-135, (supplement 1), (2016)

16. T. Cundy, E. Ackermann, E.A. Ryan, Gestational diabetes: new criteria may triple the prevalence but effect on outcomes is unclear. BMJ 348, 1567 (2014)

17. C.D. Naylor, M. Sermer, E. Chen, Sykora, K. Cesarean, Delivery in relation to birth weight and gestational glucose tolerance: pathophysiology or practice style? JAMA 275, 1165-1170 (1996)

18. R. Gorgal, E. Gonçalves, M. Barros, G. Namora, A. Magalhães, T. Rodrigues, N. Montenegro, Gestational diabetes mellitus: A risk factor for non-elective cesarean section. J. Obstet. Gynaecol. Res. 38, 154-159 (2012)

19. C. Nilsson, D. Ursing, H. Strevens, M. Landin-Olsson, Towards normalized birthweight in gestational diabetes mellitus. Acta Obstet. Gynecol. Scand. 94, 1090-1094 (2015)

20. S. Brito, A. Brett, J. Agro, Large for gestational age newborns: concept and reality. Acta Pediatr. Port. 45, 7-15 (2014)

21. T.F. Esakoff, A. Guillet, A.B. Caughey, Does small for gestational age worsen outcomes in gestational diabetics? J Matern-Fetal Neonat Med 30, 890-893 (2016) 